\title{
INTERNATIONAL
}

ECONOMIC

REVIEW

February 2002

Vol. 43, No. 1

\section{COMPETITIVE PRICING AND EFFICIENCY IN SEARCH EQUILIBRIUM*}

\author{
By Dale T. Mortensen And Randall Wright ${ }^{1}$ \\ Northwestern University and University of Pennsylvania, U.S.A.
}

\begin{abstract}
We consolidate and generalize some results on price determination and efficiency in search equilibrium. Extending models by Rubinstein and Wolinsky and by Gale, heterogeneous buyers and sellers meet according to a general matching technology and prices are determined by a general bargaining condition. When the discount rate $r$ and search costs converge to 0 , we show that prices in all exchanges are the same and equal the competitive, market clearing, price. Given positive search costs, efficiency obtains iff bargaining satisfies Hosios' condition and $r=0$. When prices are set by third-party market makers, however, we show that search equilibrium is necessarily efficient.
\end{abstract}

\section{INTRODUCTION}

The starting point for the analysis of economic exchange is the frictionless theory of competitive, market clearing, supply and demand. The purposes of the approach are to identify the determinants of trade flows and to illuminate the social role of exchange. Although this standard paradigm is a very powerful tool, there are many questions on which it sheds little light. For example, what is the process by which equilibrium prices are reached? Why does it appear that some markets, say those for labor services or housing, fail to clear? Are the claims of the theory robust to relaxation of the assumptions of perfect information and zero transactions cost? These are some of the issues that recent studies in search equilibrium have attempted to answer. The purpose of this article is to consolidate and extend some results on price determination and allocative efficiency from this literature.

\footnotetext{
* Manuscript received May 2000; revised December 2000.

${ }^{1}$ We would like to thank Kenneth Burdett, Ricardo Lagos, Derek Laing, Richard Rogerson, and two referees for very useful comments and suggestions. The NSF provided financial support. Please address correspondence to: Randall Wright, Department of Economics, University of Pennsylvania, 3718 Locust Walk, Philadelphia, PA 19104-6297. Phone: 215-898-7194. Fax: 215-573-2072. E-mail: rwright@ssc.upenn.edu.
} 
Consider the model of Rubinstein and Wolinsky (1985). In their setup, there are many homogeneous buyers and many homogeneous sellers. Each buyer wants to acquire a single unit of an indivisible good in exchange for some amount of a perfectly divisible good, and each seller has one unit of the indivisible good for sale. Agents meet bilaterally and at random according to a simple matching technology, and trade when mutually agreeable at prices given by a simple bargaining rule. Rubinstein and Wolinsky characterize equilibrium and ask whether the outcome converges to a benchmark they regard as the analog of the competitive (Walrasian, market clearing) outcome, as the rate of time preference $r$ goes to 0 . Although there are some issues about whether they adopted the right benchmark, there is no doubt that they are asking the right question: is the frictionless competitive outcome of the standard model the limit of equilibria in well-specified models with explicit frictions when these frictions get small?

Gale (1987) clarifies the benchmark one should regard as the analog to the competitive outcome and analyzes a generalized version of the model where there are perpetual potential inflows of new buyers and sellers. He maintains a very particular matching technology and bargaining solution but allows agents to be heterogeneous: different buyers (sellers) can have different valuations (costs), and each must choose whether or not to enter the market. There are typically many prices in equilibrium, depending on the buyer and seller in a given transaction. However, Gale shows that as $r$ gets small all prices tend to a common limit (a law of one price); moreover, this limit is market clearing in the sense that it equates demand and supply in terms of the flows of entering buyers and sellers. We extend these results by showing that such a law of one price holds when $r$ tends to 0 , even when there are strictly positive search costs, for any constant returns to scale matching technology, and for a generalized bargaining rule.

These extensions are interesting for the following reasons: First, we show that having positive search costs means that for all $r$ below some threshold $\widehat{r}>0$, every meeting will result in trade (when search costs are 0 we can only guarantee this in the limit when $r=0$ ). This makes the analysis relatively tractable and allows us not only to derive limiting results but to characterize the outcome rather completely, for any $r<\widehat{r}$. In particular, we can easily describe the price dispersion that arises for small but positive $r$. Second, we want to discuss efficiency, and of course, market clearing in the classical sense is not the same as efficiency when time and resources are required by the transactions process. Search equilibria are generally inefficient, as has been known since Diamond (1981) and Mortensen (1982). However, we also know from Hosios (1990) that as long as the matching technology exhibits constant returns to scale, if the bargaining rule is set just right (in terms of the matching technology) then the outcome will be efficient, at least if agents on two sides of the market are, respectively, identical.

Therefore, in order to investigate whether similar efficiency results hold here, we extend the Rubinstein-Wolinsky-Gale environment to allow a generalized matching technology and bargaining rule. Basically, the Hosios condition says that in order to maximize the aggregate gains from trade, less transaction costs, the traders' bargaining shares must reflect their marginal contribution to the value of the aggregate transaction flow. This condition is satisfied in the case of a linearly homogeneous matching technology if and only if agents' shares equal the elasticities of the matching function with respect to the stocks of buyers and sellers in the market. So in our 
model, just like the Hosios model, there is always a bargaining rule for which search equilibria are efficient. Of course, if one regards the bargaining rule as a primitive, then there is little reason to expect that in general it will satisfy Hosios' condition.

Nevertheless, Moen (1997) and Shimer (1995) have shown that there is a generalized competitive version of the search equilibrium framework that does lead to efficiency. In our interpretation of the story, one can think of third-party market makers who set up submarkets and promise a price and expected waiting time for transactions to any agents that show up. This allows buyers and sellers with different trade-offs between waiting time and price to select into appropriate submarkets. In what we call a competitive search equilibrium, each trader optimally selects a submarket, all submarkets that can attract traders are open, and all submarkets clear in the sense that they equate the inflow of participating buyers and sellers to the transaction flow. Since all gains from trade are exploited, competitive search equilibria are Pareto optimal and vice versa. Furthermore, we show that the equilibrium price in each submarket can be interpreted as the outcome of bilateral bargaining where the shares satisfy the Hosios condition. ${ }^{2}$

The rest of the article is organized as follows: Section 2 lays out the basic search equilibrium framework, discusses existence and uniqueness, and derives the limiting law of one price. Also, price dispersion is discussed for the case when $r$ is strictly positive but below some threshold, and a version of the Hosios condition for efficiency is derived. Section 3 considers competitive search equilibrium and also derives existence, uniqueness, and efficiency results. Section 4 presents some brief concluding comments.

\section{SEARCH EQUILIBRIUM}

2.1. The Basic Model. Each buyer demands one unit of an indivisible good and is willing to pay for it using another, perfectly divisible, good only if the price $p$ is less than his valuation or demand price, $x$. Each seller supplies one unit of the indivisible good only if the price exceeds his cost or supply price, $y$. Once a buyer buys or a seller sells, he leaves the market. To maintain a steady state, there is also an inflow of new participants. The potential inflow of buyers is $b$, and each entrant has a demand price drawn from a continuous distribution with c.d.f. $F(x)$; it is a potential inflow because some buyers (presumably, those with low $x$ ) may choose not to enter. Similarly, the potential inflow of sellers is $s$, and each has a supply price drawn from a continuous distribution with c.d.f. $G(y)$. The set of buyer types is given by $[\underline{x}, \bar{x}] \subseteq \Re_{+}$ and the set of seller types by $[\underline{y}, \bar{y}] \subseteq \Re_{+}$, where we assume $\bar{x}>\underline{y}$, since otherwise the market would obviously shut down. ${ }^{3}$

\footnotetext{
${ }^{2}$ One interpretation of competitive search equilibrium is that which allows search to be directed, in the sense that buyers and sellers get to choose where they go, which sorts them into appropriate submarkets. Each submarket operates like the basic search equilibrium model. In the more basic model, by contrast, search is undirected. See Acemoglu and Shimer (1999), Burdett et al. (2001), or Julien et al. (2000), e.g., for discussions of the directed search approach and other references.

${ }^{3}$ The assumption of continuous distributions of types $F$ and $G$ is a simplification that permits the use of differential methods. All the results can be extended to the case in which the distributions are discrete, given the appropriate technical fixes.
} 
Potential trading partners meet at an aggregate flow rate determined by a Pissarides (1990) style matching function $M(B, S)$, where $B$ and $S$ are the stocks of buyers and sellers currently in the market. We assume that $M$ is increasing, concave, and homogeneous of degree one. The buyer-seller ratio $B / S$, often referred to as market tightness, is denoted $\theta$. Given that matches are determined at random, the arrival rate for a seller (the rate at which he meets buyers) is $M(B, S) / S=M(\theta, 1)$, which we denote $m(\theta)$. Similarly, the arrival rate for a buyer (the rate at which he meets sellers) is $M(B, S) / B=m(\theta) / \theta$. The expected duration of time spent waiting for a match for any agent is the inverse of his arrival rate. A common exponential rate of time preference $r$ is assumed. There is also an out-of-pocket transaction or search cost per unit time, denoted $c_{b}$ for each buyer and $c_{s}$ for each seller. These search costs, plus the fact that meetings take time and agents discount, constitute the frictions in our environment.

As in Gale (1987), let $p^{*}$ and $q^{*}$ denote the competitive equilibrium price and quantity:

$$
b\left[1-F\left(p^{*}\right)\right]=s G\left(p^{*}\right)=q^{*}
$$

They are competitive in the sense that they are market clearing: the first equality equates the flow into the market of buyers to that of sellers, and these inflows equal the outflow, or the quantity transacted $q^{*}$, in steady state. However, the terms of trade in our market are not generally given by (1) but are naturally determined by bilateral bargaining. While there are a variety of ways in which to proceed, we assume here that when a buyer and seller meet, one of the two, chosen at random, announces a take-it-or-leave-it price offer. Let $\beta$ be the probability the seller makes the offer. If an offer is rejected, the agents part and continue searching as though they never met; if the offer is accepted, exchange occurs and they exit the market.

In fact, here this bargaining protocol is equivalent to the generalized Nash solution. To see this, let $V_{b}(x)$ be the value function from participating in the market for a buyer with demand price $x$ and let $V_{s}(y)$ be the analog for a seller with supply price $y$. A take-it-or-leave-it price offer $p$ is acceptable to buyer $x$ if and only if $x-p \geq V_{b}(x)$, and is acceptable to seller $y$ if and only if $p-y \geq V_{s}(y)$. Given complete information, the optimal strategy of any agent choosing the price is to offer the other party's reservation value; i.e., $p_{b}(y)=y+V_{s}(y)$ when the buyer sets the price knowing that the seller is of type $y$ and $p_{s}(x)=x-V_{b}(x)$ when the seller sets the price knowing that the buyer is of type $x$. The expected price in a transaction between buyer $x$ and seller $y$ is given by $p(x, y)=\beta p_{s}(x)+(1-\beta) p_{b}(y)$. Inserting $p_{s}(x)=x-V_{b}(x)$ and $p_{b}(y)=y+V_{s}(y)$ and rearranging, we have

$$
p(x, y)=y+V_{s}(y)+\beta\left[x-y-V_{b}(x)-V_{s}(y)\right]
$$

Hence, one can interpret the outcome as the generalized Nash solution to bargaining over the joint trade surplus, $x-y-V_{b}(x)-V_{s}(y)$, where $\beta$ is the bargaining power of the seller and the threat points are given by $V_{b}(x)$ and $V_{s}(y){ }^{4}$

\footnotetext{
${ }^{4}$ As is well known, the generalized Nash solution is equivalent to an alternating-offer bargaining game when the time between offers vanishes; see Osborne and Rubinstein (1990) for example.
} 
Given $p(x, y)$, we can describe payoffs and the set of agents who enter the market as follows: The expected value of participation for a buyer with valuation $x$ in steady state solves

$$
r V_{b}(x)=\frac{m(\theta)}{\theta} \int \max \left\{x-p(x, y)-V_{b}(x), 0\right\} d \Gamma(y)-c_{b}
$$

where $\Gamma(y)$ is the distribution of seller types in the market, which, in general, differs from the exogenous distribution of potential entrants $G(y)$ since not everyone necessarily enters. Analogously, for a seller with cost $y$,

$$
r V_{s}(y)=m(\theta) \int \max \left\{p(x, y)-y-V_{s}(y), 0\right\} d \Phi(x)-c_{s}
$$

where $\Phi(x)$ is the distribution of buyer types in the market. Inserting the bargaining solution (2) into (3) and (4), we have

$$
\begin{aligned}
& r V_{b}(x)+c_{b}=\frac{m(\theta)(1-\beta)}{\theta} \int \max \left\{x-y-V_{b}(x)-V_{s}(y), 0\right\} d \Gamma(y) \\
& r V_{s}(y)+c_{s}=m(\theta) \beta \int \max \left\{x-y-V_{b}(z)-V_{s}(y), 0\right\} d \Phi(x)
\end{aligned}
$$

One can easily verify that $V_{b}(x)$ is increasing and $V_{s}(y)$ decreasing. Hence, only buyers with $x \geq R_{b}$ and sellers with $y \leq R_{s}$ enter the market, where

$$
V_{b}\left(R_{b}\right)=0=V_{s}\left(R_{s}\right)
$$

The pair $\left(R_{b}, R_{s}\right)$ identifies the marginal participating types.

We now derive the steady-state conditions. First note that in any meeting, exchange takes place if and only if the gain from trade is no less than the sum of the values of continued search, $x-y \geq V_{b}(x)+V_{s}(y)$. Hence, for buyers with $x \geq R_{b}$, we can equate the flow into the market and the flow out to get

$$
b d F(x)=\frac{m(\theta)}{\theta} B d \Phi(x) \int I\left[x-y-V_{b}(x)-V_{s}(y)\right] d \Gamma(y)
$$

where $I(z)$ is an indicator function that takes on the value 1 if $z \geq 0$ and 0 otherwise. ${ }^{5}$ Of course, for buyers with $x<R_{b}$, who never enter the market, the steady-state condition is $d \Phi(x)=0$. Similarly, for sellers with $y \leq R_{s}$, we have

$$
s d G(y)=m(\theta) S d \Gamma(y) \int I\left[x-y-V_{b}(x)-V_{s}(y)\right] d \Phi(x)
$$

and for sellers with $y>R_{s}$, we have $d \Gamma(y)=0$.

Integrating both sides of (8) over $\left[R_{b}, \bar{x}\right]$ yields

$$
b\left[1-F\left(R_{b}\right)\right]=\frac{m(\theta)}{\theta} B \iint I\left[x-y-V_{b}(x)-V_{s}(y)\right] d \Gamma(y) d \Phi(x)
$$

\footnotetext{
${ }^{5}$ The flow in is $b d F(x)$ since $d F(x)$ is the density of potential entrants, and hence the density of actual entrants for all $x \geq R_{b}$. To compute the flow out, note that there are $B d \Phi(x)$ type $x$ buyers in the market in steady state, they meet sellers at rate $m(\theta) / \theta$, and a meeting results in exchange with probability given by the integral.
} 
Similarly, intergrating (9) over $\left[\underline{y}, R_{s}\right]$ yields

$$
s G\left(R_{s}\right)=m(\theta) S \iint I\left[x-y-V_{b}(x)-V_{s}(y)\right] d \Gamma(y) d \Phi(x)
$$

Since $\operatorname{Bm}(\theta) / \theta=\operatorname{Sm}(\theta)=M(S, B),(10)$ and (11) imply

$$
b\left[1-F\left(R_{b}\right)\right]=s G\left(R_{s}\right)=M(S, B) \iint I\left[x-y-V_{b}(x)-V_{s}(z)\right] d \Phi(x) d \Gamma(y)
$$

This simply says that the total flow of buyers into the market equals the total flow of sellers into the market, and both equal the total flow out of the market.

A (steady-state) search equilibrium can now be defined in terms of the marginal participating types $\left(R_{b}, R_{s}\right)$, the stocks of buyers and sellers in the market $(B, S)$, and the distribution functions over types in the market $[\Phi(x), \Gamma(y)]$, satisfying the participation conditions in (7) and steady-state conditions in (12), (8), and (9). Other variables, such as the price function $p(x, y)$ or market tightness $\theta$, which are also implicitly part of an equilibrium, can easily be recovered from the above conditions. In general, the existence problem involves finding a fixed point in the space of distribution functions $[\Phi(x), \Gamma(y)]$. Rather than dwell on the technicalities required to establish a general existence result, we focus in the sequel on the relatively simple case that arises when $r$ is small.

2.2. The Law of One Price: $r=0$. In the limiting case when $r \rightarrow 0$ the left-hand side of (5) does not depend on $x$, and so neither does the right-hand side. This means that $x-V_{b}(x)=k$ for some $k$ that is independent of $x$ for everyone in the market. In particular, for the marginal participating buyer, we have $R_{b}-V_{b}\left(R_{b}\right)=k$, and since $V_{b}\left(R_{b}\right)=0$ we know $k=R_{b}$. A symmetric argument applies to sellers. Hence,

$$
x-V_{b}(x)=R_{b} \quad \forall x \geq R_{b} \quad \text { and } \quad y+V_{s}(y)=R_{s} \quad \forall y \leq R_{s}
$$

Substituting these into (5) and (6), given $r=0$, we have

$$
(1-\beta) \max \left\{R_{b}-R_{s}, 0\right\}=\frac{c_{b} \theta}{m(\theta)} \quad \text { and } \quad \beta \max \left\{R_{b}-R_{s}, 0\right\}=\frac{c_{s}}{m(\theta)}
$$

This implies that $R_{b}>R_{s}$ as long as $c_{b}>0$ and $c_{s}>0$. As a consequence the gains from trade exceed the sum of the values of continued search for every participating pair:

$$
x-y-V_{b}(x)-V_{s}(y)=R_{b}-R_{s}=\frac{c_{b} \theta+c_{s}}{m(\theta)}>0 \quad \forall x \geq R_{b}, \quad y \leq R_{s}
$$

Therefore, at least in the limiting case where $r \rightarrow 0$, every meeting results in trade. ${ }^{6}$

Given that every meeting results in trade, (12) reduces to

$$
b\left[1-F\left(R_{b}\right)\right]=s G\left(R_{s}\right)=M(B, S)
$$

\footnotetext{
${ }^{6}$ Since the inequality in (15) is strict, continuity actually implies that all meetings result in trade not only in the limit when $r \rightarrow 0$ but also for all $r$ below some strictly positive threshold $\widehat{r}$, at least as long as $c_{b}, c_{s}>0$ (see below).
} 
Moreover, the endogenous distribution functions $[\Phi(x), \Gamma(y)]$ are now simple. For instance, (9) becomes $s d G(y)=M(B, S) d \Gamma(y)=s G\left(R_{s}\right) d \Gamma(y)$; hence, $d \Gamma(y)=$ $d G(y) / G\left(R_{s}\right)$. Similarly, $d \Phi(x)=d F(x) /\left[1-F\left(R_{b}\right)\right]$. Equilibrium is now given by the triple $\left(\theta, R_{b}, R_{s}\right)$ solving the two conditions in (14) and the first equation of (16). Note that (14) implies $c_{b} \theta /(1-\beta)=\left(R_{b}-R_{s}\right) m(\theta)=c_{s} / \beta$, and so we can first solve for market tightness,

$$
\theta=\frac{c_{s}}{c_{b}} \frac{\beta}{1-\beta}
$$

before knowing $R_{s}$ and $R_{b}$. Then, given $\theta$, (15) implies

$$
R_{b}-R_{s}=K
$$

where $K=\left[c_{b} \theta+c_{s}\right] / m(\theta)$.

Although we give a more general existence result below, it is worth first discussing the issue when things are relatively simple. First, (18) defines an increasing relationship while (16) defines a decreasing relationship between $R_{b}$ and $R_{s}$, as seen in Figure 1 . Hence, there is at most one pair $\left(R_{b}, R_{s}\right)$ solving these conditions. As is clear from the figure, if $K=0$, there is a solution $R_{b}=R_{s}=R$, where $\underline{y}<R<\bar{x}$ (given our maintained assumption $\underline{y}<\bar{x}$ ); this means that a positive measure of agents enters on both sides of the market, and so we say the market is active, or open. Indeed, for all $K<\bar{x}-\underline{y}$ there exists a solution with $R_{b}<\bar{x}$ and $R_{s}>\underline{y}$, but as soon as $K$ exceeds $\bar{x}-y$ there is no solution with $R_{b}<\bar{x}$ and $R_{s}>y$, and the market shuts down. To understand the role of $K$, suppose we fix the ratio $\bar{c}_{s} / c_{b}$, which means $\theta$ is

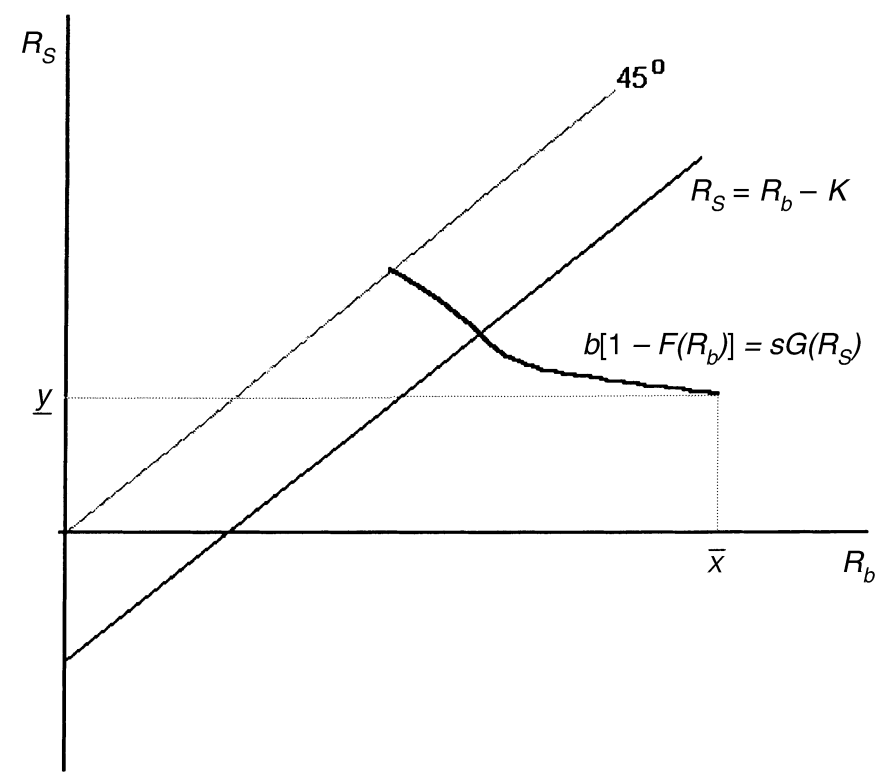

FIGURE 1 
fixed by (17). Then $K$ is proportional to $c_{s}\left(\right.$ or $\left.c_{b}\right)$. For small search costs the market is open, but as these costs rise it eventually closes.

To describe prices, substitute (13) into (2) to yield

$$
p(x, y)=\widehat{p}=\beta R_{b}+(1-\beta) R_{s} \quad \forall(x, y)
$$

Therefore, in the limit as $r \rightarrow 0$ the price actually does not depend on the types in the meeting $(x, y)$. This generalizes the result found by Gale (1987), who derives this limiting law of one price for $\beta=1 / 2, c_{b}=c_{s}=0$, and a special matching technology. The equilibrium with $r \rightarrow 0$ is illustrated in Figure 2. As in the standard supply and demand diagram, the vertical axis represents price $p$; the horizontal is quantity $q$; the curve $D D$ is the graph of the inverse demand function, which in our case is $F^{-1}(1-q / b)$; and the curve $S S$ is the graph of the inverse supply function, which is $G^{-1}(q / s)$. The competitive market clearing price-quantity pair $\left(p^{*}, q^{*}\right)$ occurs at the intersection of the two curves.

In search equilibrium the transaction flow $\widehat{q}=M(B, S)=s G\left(R_{s}\right)=b\left[1-F\left(R_{b}\right)\right]$ is the value of $q$ such that the vertical difference between the $D D$ and $S S$ curves equals $\left(c_{b} \theta+c_{s}\right) / m(\theta)$, since this is what $R_{b}-R_{s}$ must equal by (15). As seen in Figure 2, the competitive market clearing price $p^{*}$ is necessarily bracketed by $R_{s}$ and $R_{b}$. The search equilibrium price $\widehat{p}=\beta R_{b}+(1-\beta) R_{s}$ is a simple average of these values. Consequently, as the participation costs $c_{b}$ and $c_{s}$ vanish holding their ratio constant, since $R_{s}$ and $R_{b}$ converge to the same limit $R$, we see that $(\widehat{p}, \widehat{q})$ converges to the competitive market clearing pair $\left(p^{*}, q^{*}\right)$. We conclude that when frictions become small in the sense that $r, c_{b}$, and $c_{s}$ vanish, the search equilibrium outcome converges



FIGURE 2 
to the competitive outcome. Note that this is independent of relative bargaining power, as reflected in the value of $\beta .^{7}$

2.3. Price Dispersion: $r>0$. As mentioned above, when $c_{b}, c_{s}>0$, it follows from (15) that every meeting results in exchange for all $r$ below some threshold $\widehat{r}$, and not only in the limit at $r \rightarrow 0$. Given $r$ is strictly positive and less than $\widehat{r}$, the outcome is still relatively easy to characterize and is interesting precisely because the law of one price does not hold. To describe the outcome, begin by rewriting the value functions in (5) and (6) as follows: ${ }^{8}$

$$
V_{b}(x)=\frac{(1-\beta) m(\theta)}{(1-\beta) m(\theta)+r \theta}\left(x-R_{b}\right) \quad \text { and } \quad V_{s}(y)=\frac{\beta m(\theta)}{\beta m(\theta)+r}\left(R_{s}-y\right)
$$

Substitution of (20) into (2) yields

$$
p(x, y)=\beta\left[\frac{r \theta x+(1-\beta) m(\theta) R_{b}}{r \theta+(1-\beta) m(\theta)}\right]+(1-\beta)\left[\frac{r y+\beta m(\theta) R_{s}}{\beta m(\theta)+r}\right]
$$

Hence, price increases with the buyer's desire for the good, $x$, and with the seller's cost of production, $y$.

The next thing to do is to find the value of the threshold $\widehat{r}$ below which all meetings result in trade. To this end, begin by substituting from (20) back into (5) and rearranging to get

$$
\frac{c_{b} \theta}{m(\theta)}=(1-\beta) \int\left[R_{b}-\frac{r y+\beta m(\theta) R_{s}}{r+\beta m(\theta)}\right] d \Gamma(y)
$$

Using the steady-state condition $d \Gamma(y)=d G(y) / G\left(R_{s}\right)$, we have

$$
\frac{c_{b} \theta}{m(\theta)}=(1-\beta) \int\left[R_{b}-\frac{r y+\beta m(\theta) R_{s}}{r+\beta m(\theta)}\right] \frac{d G(y)}{G\left(R_{s}\right)}
$$

Similarly, substituting from (20) back into (6) and rearranging, we have

$$
\frac{c_{s}}{m(\theta)}=\beta \int\left[-R_{s}+\frac{r \theta x+(1-\beta) m(\theta) R_{b}}{r \theta+(1-\beta) m(\theta)}\right] \frac{d F(x)}{1-F\left(R_{b}\right)}
$$

These two equations together with the market clearing condition (16) determine $\left(\theta, R_{b}, R_{s}\right)$. A unique solution exists if $r, c_{b}$, and $c_{s}$ are small enough, as we detail in Proposition 3.

\footnotetext{
${ }^{7}$ Furthermore, the steady-state ratio of the stock of buyers to the stock of sellers in the market waiting to trade tends to 0 as the market structure tends to monopsony $(\beta \rightarrow 1)$ and to $\infty$ as the market structure tends to monopsony $(\beta \rightarrow 0)$; hence, one can say that the relative number of traders on the two sides of the market is determined by bargaining power, rather than vice versa.

${ }^{8}$ To derive $V_{b}(x)$, differentiate $(5)$ to get $V_{b}^{\prime}(x)=(1-\beta) m /[r \theta+(1-\beta) m]$, which is constant, so $V_{b}(x)$ is linear. Using $V_{b}\left(R_{b}\right)=0$, we can determine the intercept. A similar argument yields $V_{s}(y)$.
} 
Given an equilibrium exists, we know that every meeting results in exchange if and only if $x-y-V_{b}(x)-V_{s}(y) \geq 0$ for all participants in the market, i.e., for all $x \geq R_{b}$ and $y \leq R_{s}$. It is straightforward to verify using (20) that this holds if and only if $R_{b} \geq R_{s}$. Hence, the critical $\widehat{r}$ is that for which $R_{b}=R_{s}$. However, $R_{b}=R_{s}$ means that their common value is the competitive price $p^{*}$, since $p^{*}$ is bracketed by $R_{b}$ and $R_{s}$, where $p^{*}$ can be regarded as a parameter here since it depends only on the exogenous components $b, s, F$, and $G$. Inserting $R_{b}=R_{s}=p^{*}$ into (22) and (23), we solve each equation for $r$ and then equate the solutions. The result is

$$
\begin{aligned}
r & =\frac{\beta c_{b} \theta}{(1-\beta)\left[p^{*}-\int_{\underline{y}}^{p^{*}} \frac{y d G(y)}{G\left(p^{*}\right)}-\frac{c_{b} \theta}{(1-\beta) m(\theta)}\right]} \\
& =\frac{(1-\beta) c_{s}}{\beta \theta\left[\int_{p^{*}}^{\bar{x}} \frac{x d F(x)}{1-F\left(p^{*}\right)}-p^{*}-\frac{c_{s}}{\beta m(\theta)}\right]}
\end{aligned}
$$

Note that

$$
p^{*}-\int_{\underline{y}}^{p^{*}} \frac{y d G(y)}{G\left(p^{*}\right)}>0 \quad \text { and } \quad \int_{p^{*}}^{\bar{x}} \frac{x d F(x)}{1-F\left(p^{*}\right)}-p^{*}>0
$$

given the assumption of both buyer and seller diversity. Also, by assumption, $m(\theta)$ is increasing and strictly concave and $\theta / m(\theta) \rightarrow 0$ as $\theta \rightarrow 0$. These facts imply that the right-hand side of (24) is strictly increasing in $\theta$ and equals zero at $\theta=0$, while the right-hand side of (25) is strictly decreasing and converges to infinity as $\theta \rightarrow 0$. Consequently, there exists a unique solution $\widehat{\theta}>0$ to the second equality; this is the unique value for market tightness such that the market clearing condition (16) holds when $R_{b}=R_{s}=p^{*}$. Finally, associated with $\widehat{\theta}$ we get the critical discount rate $\widehat{r}$ from (24). In summary, all meetings result in trade if and only if $r<\widehat{r}$ where $\widehat{r}>0$ as long as $c_{b}, c_{s}>0$, and even though we have price dispersion the model is still quite tractable because of the fact that every meeting results in trade. ${ }^{9}$

2.4. Efficiency. We have seen that search equilibrium is approximately market clearing when frictions are small, for any value of the bargaining power parameter $\beta$. However, it has been understood since Diamond (1981) and Mortensen (1982) that search equilibria are not generally efficient. The reason is that a trader's welfare is inversely related to the time required to make an exchange, except in the limiting

${ }^{9}$ Note that $p^{*} \in[\underline{x}, \bar{x}] \cap[\underline{y}, \bar{y}]$ implies

$$
\int_{p^{*}}^{\bar{x}} \frac{x d F(x)}{1-F\left(p^{*}\right)}-p^{*} \rightarrow 0
$$

as the support of the distribution of buyer types collapses to a point, and similarly

$$
p^{*}-\int_{\underline{y}}^{p^{*}} \frac{y d G(y)}{G\left(p^{*}\right)} \rightarrow 0
$$

as seller dispersion vanishes. Hence, $\widehat{r} \rightarrow \infty$ in either case. In other words, if either all buyers are identical or all sellers are identical, then all meetings result in trade for any value of $r$. 
case of zero frictions, and these waiting times depend nontrivially on the behavior of other agents. Although inefficiency characterizes search equilibrium for an arbitrary $\beta$, when the matching function is homogeneous of degree one and all buyers and sellers are, respectively, identical, Hosios (1990) shows that a social optimum is achieved if $\beta$ is set equal to the elasticity of the matching function with respect to sellers. For this value of $\beta$ the two search externalities-the congestion effect imposed by every other agent on the same side of the market and the thick market effect attributable to the participation of agents on the other side-exactly offset.

In the case of a zero discount rate and a linear homogeneous matching function, it is easy to see that Hosios' result also holds here even though we have heterogeneous traders. One can think of the social planner as choosing the best among the set of search equilibria parameterized by $\beta .{ }^{10}$ Given the assumption of linear preferences, the appropriate welfare criterion is the steady-state gains from trade realized by buyers and sellers, less the flow search cost, per period. In other words, the planner's problem is

$$
\max _{\beta \in(0,1)} b \int_{R_{b}}^{\bar{x}}(x-\widehat{p}) d F(x)+s \int_{\underline{y}}^{R_{s}}(\widehat{p}-y) d G(y)-c_{b} B-c_{s} S
$$

subject to conditions that $\left(R_{b}, R_{S}\right)$ and $(B, S)$ satisfy the above equilibrium conditions and the price $\widehat{p}$ is given by (19).

Note that the integral in (26) is consumer plus producer surplus over all values of $q$ out to the equilibrium trade flow $\widehat{q}=M(B, S)$. As total search cost satisfies

$$
c_{b} B+c_{s} S=\left[\frac{c_{b} \theta+c_{s}}{m(\theta)}\right] M(B, S)=\left[R_{b}-R_{s}\right] \widehat{q}
$$

by virtue of the homogeneity of the matching function and the equilibrium condition (18), the optimal choice of $\beta$ is that which maximizes aggregate surplus net of transactions cost, indicated by the shaded area in Figure 2. But maximizing the shaded area is equivalent to minimizing the search cost incurred by both parties per trade, equal in equilibrium to the difference $R_{b}-R_{s}$. As the necessary and sufficient condition is

$$
\frac{m^{\prime}(\theta)}{m(\theta)-\theta m^{\prime}(\theta)}=\frac{c_{b}}{c_{s}}
$$

a comparison with (17) verifies that the optimal choice of the seller's share parameter is uniquely determined by the Hosios condition

$$
\beta=1-\frac{\theta m^{\prime}(\theta)}{m(\theta)}=\frac{S M_{s}(B, S)}{M(B, S)}
$$

2.5. Three Results. Here we summarize the main results of this section.

\footnotetext{
${ }^{10}$ This formulation of the planner's problem would be too restrictive in the case of a matching function that is not homogeneous of degree one, because then the optimal solution is not supported by any search equilibrium.
} 
Proposition 1 (Existence And Uniqueness). Given $\left(c_{b}, c_{s}\right)>0$, a unique steady-state search equilibrium exists for all $r \in(0, \widehat{r}]$ with the property that every participating buyer-seller pair trades when they meet, where $\widehat{r}$ satisfies (24).

Proof. Basically, we need to generalize the argument behind Figure 1 to the case $r>0$. Under the hypothesis $r \leq \widehat{r}$, every participating buyer-seller pair trades because $R_{b} \geq R_{s}$ by construction. Then an equilibrium can be characterized by the triple $\left(\theta, R_{b}, R_{s}\right)$ that satisfies equations (16), (22), and (23). By (16), $R_{s}$ is a decreasing function of $R_{b}$,

$$
R_{s}=\rho\left(R_{b}\right)=G^{-1}\left(\frac{b}{s}\left[1-F\left(R_{b}\right)\right]\right)
$$

Using this to eliminate $R_{s}$ in (22) and (23), one obtains

$$
\begin{aligned}
\frac{c_{b} \theta}{(1-\beta) m(\theta)} & =\int_{\underline{y}}^{\rho\left(R_{b}\right)}\left[R_{b}-\frac{r y+\beta m(\theta) \rho\left(R_{b}\right)}{r+\beta m(\theta)}\right] \frac{d G(y)}{G\left[\rho\left(R_{b}\right)\right]} \\
\frac{c_{s}}{\beta m(\theta)} & =\int_{R_{b}}^{\bar{x}}\left[-\rho\left(R_{b}\right)+\frac{r \theta x+(1-\beta) m(\theta) R_{b}}{r \theta+(1-\beta) m(\theta)}\right] \frac{d F(x)}{1-F\left(R_{b}\right)}
\end{aligned}
$$

The right-hand side of either equation is strictly increasing in $R_{b}$. The left-hand side of the first equation is strictly increasing while the right-hand side of the second is strictly decreasing in $\theta$. Hence the first equation defines a strictly increasing relation between the two variables while the second defines a strictly decreasing relation. Therefore, if a nonnegative solution $\left(R_{b}, \theta\right)$ exists, it is unique.

The final step is to show that there is a positive solution. Since $R_{b} \geq R_{s}$ we have consequently, $R_{b} \geq p^{*} \geq R_{s}=\rho\left(R_{b}\right)$ by the definition of $p^{*}$, where $p^{*}>0$. Hence, the solution for $\theta$ must lie between $\theta_{L}$, defined as the solution to the first equation when $R_{b}=p^{*}=R_{s}$, and $\theta_{H}$, defined as the solution to the second equation when $R_{b}=p^{*}=R_{s}$. Inserting $R_{b}=p^{*}$ we have

$$
\begin{aligned}
\frac{c_{b} \theta}{(1-\beta) m} & =r \int_{\underline{y}}^{p^{*}}\left[\frac{p^{*}-y}{r+\beta m}\right] \frac{d G(y)}{G\left(p^{*}\right)} \\
\frac{c_{s}}{\beta m} & =r \theta \int_{p^{*}}^{\bar{x}}\left[\frac{x-p^{*}}{r \theta+(1-\beta) m}\right] \frac{d F(x)}{1-F\left(p^{*}\right)}
\end{aligned}
$$

As the right-hand side of both equations is positive, by assumption, so are $\theta_{L}$ and $\theta_{H}$. Consequently, there is a positive solution for $\theta$.

Proposition 2 (The LAw of One Price). Consider any sequence of search equilibria defined by a sequence of strictly positive triples $\left(r, c_{b}, c_{s}\right)$ converging to $(0,0,0)$. The expected price of an exchange between any participating pair $p(x, y)$ converges to the competitive equilibrium price $p^{*}$ in the limit.

Proof. As the triple $\left(r, c_{b}, c_{s}\right)>0$ tends to zero, it is clear from the above discussion that $R_{b}$ and $R_{s}$ converge to each other (independent of the order of limits), and their common limit is $p^{*}$. Hence, the result is implied by taking the limit in (21). 
Proposition 3 (EFFICIENCY). For $r=0$, search equilibrium maximizes the expected present value of the future flow of aggregate trade surplus less search costs if and only if $\beta$ equals the elasticity of the matching function with respect to $S$.

Proof. Clear from (28).

\section{COMPETITIVE SEARCH EQUILIBRIUM}

Although Proposition 3 says that search equilibrium is efficient when the gains from trade are split in a particular way, there is no reason to expect that bilateral bargaining should necessarily deliver the correct outcome. That is, if the bargaining solution is taken as a primitive, equilibria will not generally be efficient. However, Moen (1997) and Shimer (1995) demonstrate that Hosios' condition does obtain in extended formulations of the model in which price offers and waiting times are known prior to the matching process. Here we interpret their equilibrium construct as a complete market equilibrium in which the expected duration until a trade is executed will be implicitly priced, and extend the result to the case of heterogeneity on both sides of the market.

Because search externalities are internalized in this competitive formulation of search equilibrium, participation and sorting of buyers and sellers into a collection of submarkets that offer different expected waiting times are Pareto efficient and, conversely, any Pareto efficient solution to the participation and sorting problem can be interpreted as a competitive search equilibrium. In the case of a zero rate of discount, the competitive search equilibrium is the search equilibrium with shares that satisfy the Hosios condition. More generally, the equilibrium pricing across submarkets can be formally interpreted as the particular bilateral bargaining outcome in each submarket for which the Hosios condition holds. Finally, we will show that there is only one competitive search equilibrium for all positive sufficiently small rates of discount, and that equilibrium necessarily maximizes the aggregate values of buyer and seller participation.

3.1. The Basic Model. Imagine that each trader gets to choose to participate in one of a collection $\Theta$ of submarkets. In each submarket, buyers and sellers meet randomly at rates determined by the number of agents who participate. Since the meeting rates, $m(\theta)$ for sellers and $m(\theta) / \theta$ for buyers, are uniquely determined by market tightness $\theta$ in a submarket, the set of submarkets $\Theta$ is the set of distinct values for the market-tightness parameter offered by the market. Differences in trading prices across the submarkets, given by the endogenous price function $p: \Theta \rightarrow \Re_{+}$, implicitly price differences in expected waiting times. So each submarket is characterized by a pair $(p, \theta)$, and each participating trader selects the most preferred submarket taking this price function and the set of submarkets as given.

An equilibrium price function is market clearing if the steady-state number of participating buyers and sellers in each submarket adjusts to equate the flow of buyers and sellers into each submarket with the transactions outflow. That is, taking $(p, \theta)$ as given, buyers and sellers participate in sufficient numbers to generate the 
correct value of $\theta$ endogenously in this submarket. The equilibrium set of markets $\Theta$ satisfies two conditions: (i) each submarket is open, in the sense that it is the preferred choice of some buyer and some seller type; and (ii) markets are complete, in the sense that there is no other potential submarket $(p, \theta)$ that would be preferred by a buyer and seller pair to any of those that are open.

Every meeting in a submarket results in trade. Hence, (3) and (4) imply that the type-contingent stationary values of buyers and sellers in submarket $(p, \theta)$ are

$$
\begin{aligned}
U_{b}(x, p, \theta) & =\frac{m(\theta)(x-p)-\theta c_{b}}{r \theta+m(\theta)} \\
U_{s}(y, p, \theta) & =\frac{m(\theta)(p-y)-c_{s}}{r+m(\theta)}
\end{aligned}
$$

Given any price function $p(\theta)$, the values of participation are equal to the values of choosing the preferred submarket; i.e.,

$$
V_{b}(x)=\max _{\theta \in \Theta} U_{b}[x, p(\theta), \theta] \quad \text { and } \quad V_{s}(y)=\max _{\theta \in \Theta} U_{s}[y, p(\theta), \theta]
$$

The value of buyer participation increases with $x$ and the value of seller participation decreases with $y$. Hence, all buyers with $x \geq R_{b}$ and all sellers with $y \leq R_{s}$ participate, where the marginal types again solve

$$
V_{b}\left(R_{b}\right)=V_{s}\left(R_{s}\right)=0
$$

Let $\gamma_{b}(x, \theta)$ and $\gamma_{s}(y, \theta)$ represent an assignment of buyers and sellers, equal, respectively, to the fraction of the entering flow of each type assigned to the submarket indexed by $\theta$. A market clearing assignment equates the flows of participating buyers and sellers into each submarket $\theta$ :

$$
b \int \gamma_{b}(x, \theta) d F(x)=s \int \gamma_{s}(y, \theta) d G(y) \quad \forall \theta \in \Theta
$$

where $\int_{\Theta} \gamma_{b}(x, \theta) d \theta=\int_{\Theta} \gamma_{s}(y, \theta) d \theta=1 \forall x \geq R_{b}$ and $y \leq R_{s}$. An assignment is called competitive if and only if it is market clearing and respects the submarket selfselection and participation conditions (31) and (32):

$$
\begin{aligned}
& \gamma_{b}(x, \theta)=0 \quad \text { if either } x<R_{b} \quad \text { or } \quad \theta \notin \arg \max U_{b}[x, p(\theta), \theta] \quad \forall x \geq R \\
& \gamma_{s}(x, \theta)=0 \quad \text { if either } y>R_{s} \text { or } \theta \notin \arg \max U_{s}[y, p(\theta), \theta] \quad \forall y \leq R_{s}
\end{aligned}
$$

Given this, we can formalize our openness and completeness conditions as follows. When we say that each market in $\Theta$ is open, we mean that it attracts some buyers and sellers: $\forall \theta \in \Theta \exists(x, y) \in\left[R_{b}, \bar{x}\right] \times\left[\underline{y}, R_{s}\right]$ such that

$$
\theta=\arg \max U_{b}[x, p(\theta), \theta]=\arg \max U_{s}[y, p(\theta), \theta]
$$

When we say $\Theta$ is complete we mean there is no possible submarket that is not open but is strictly preferred by some buyers and sellers: we cannot find a $\theta \notin \Theta$, a $p \in[\underline{y}, \bar{x}]$, and a pair $(x, y) \in[\underline{x}, \bar{x}] \times[\underline{y}, \bar{y}]$ such that

$$
U_{b}(x, p, \theta)>V_{b}(x) \quad \text { and } \quad U_{s}(y, p, \theta)>V_{s}(y)
$$

One way to interpret these conditions is to invoke the existence of third-party market makers with a profit motive who can set up submarkets. If there are positive 
but sufficiently small costs of opening a submarket, anything that failed to attract participants would not exist, while those that could attract both buyers and sellers from existing markets would be created. ${ }^{11}$ In any case, a competitive search equilibrium is a pair of marginal participant types $\left(R_{s}, R_{b}\right)$, an assignment $\left[\gamma_{b}(x, \theta), \gamma_{s}(y, \theta)\right]$, a set $\Theta$, and a price function $p: \Theta \rightarrow \Re_{+}$that satisfy (32)-(37).

3.2. The Equilibrium Price Function. In the above formulation, the set of steady-state equilibrium competitive assignments is equivalent to the set of Pareto efficient assignments of buyers and sellers to submarkets. Specifically, in $(\theta, p)$ space, buyers and sellers have indifference curves along which $U_{b}(x, p, \theta)$ and $U_{s}(y, p, \theta)$ are constants, with marginal rates of substitution given by

$$
\begin{aligned}
& \left.\frac{d p}{d \theta}\right|_{U_{b}}=\frac{\left[\theta m^{\prime}(\theta)-m(\theta)\right]\left[r(x-p)+c_{b}\right]}{m(\theta)[r \theta+m(\theta)]} \\
& \left.\frac{d p}{d \theta}\right|_{U_{s}}=\frac{-m^{\prime}(\theta)\left[r(p-y)+c_{s}\right]}{m(\theta)[r+m(\theta)]}
\end{aligned}
$$

which we get by differentiating (29) and (30). Both are downward sloping in $(\theta, p)$ space. For any buyer-seller pair $(x, y)$ in the same submarket $\theta$, given $p(\theta)$, it must be the case that these marginal rates of substitution are equal to $p^{\prime}(\theta)$, since otherwise the completeness condition would be violated.

Equating the marginal rates of substitution in (38) and (39) yields a somewhat complicated expression, but it can be rewritten as

$$
p=\left[1-\frac{\theta m^{\prime}(\theta)}{m(\theta)}\right]\left[x-V_{b}(x)\right]+\frac{\theta m^{\prime}(\theta)}{m(\theta)}\left[y+V_{s}(y)\right]
$$

$\forall \theta \in \Theta$ and $(x, y)$ such that $\gamma_{b}(x, \theta)$ and $\gamma_{s}(y, \theta)>0$. This is equivalent to the general pricing rule for search equilibrium given above, condition (2), with the seller's share parameter $\beta$ set equal to the elasticity $\theta m^{\prime}(\theta) / m(\theta)$. In other words, the price in every submarket can be viewed as the bilateral bargaining outcome for which the Hosios condition holds. In particular, when $r=0$, equating (38) and (39) yields

$$
\frac{c_{b}}{c_{s}}=\frac{m^{\prime}(\theta)}{m(\theta)-\theta m^{\prime}(\theta)}
$$

which is condition (27) for minimizing transaction cost.

When $r=0$, the marginal rates of substitution are the same for all buyers and sellers (i.e., independent of $x$ and $y$ ). In this case, all buyers and sellers participate in a single market with a price that induces optimal participation via the Hosios condition. In other words, the economy looks exactly like the basic search model in the previous section with $\beta$ set optimally. More generally, when $r>0$, an equilibrium $p(\theta)$ is a fixed point of the map defined by (40) together with an assignment of types to submarkets. Below we show that equilibrium is unique at least for small $r$. We first describe the way the agents select into different submarkets. Intuitively, the marginal rate of substitution between $p$ and $\theta$ decreases with $y$ for sellers and increases with $x$ for

\footnotetext{
${ }^{11}$ Greenwald and Stiglitz (1988) tell a similar story.
} 
buyers, and as a consequence sellers with higher $y$ choose submarkets where the buyers have higher $x$. The price in a submarket visited by buyers with higher $x$ and sellers with higher $y$ is greater. At the same time, market tightness in such a submarket must be lower, because buyers are only willing to accept higher $p$ for a higher arrival rate, while sellers will only accept a lower arrival rate in exchange for higher $p$.

To make the point more precisely, consider Figure 3. The curve $E E$ represents a candidate equilibrium price function, $p(\theta)$, which is downward sloping because the indifference curves of all buyers and sellers are. Specifically, consider the point $\left(p^{\prime}, \theta^{\prime}\right)$ defined by $p^{\prime}=p\left(\theta^{\prime}\right)$. Suppose it is the preferred point for buyers with demand price $x^{\prime}$ and sellers with supply price $y^{\prime}$ over the set of submarkets. As a necessary condition for optimality, the buyer's indifference curve through the point, labeled $I_{b}^{\prime} I_{b}^{\prime}$, and the seller's indifference curve through the same point, labeled $I_{s}^{\prime} I_{s}^{\prime}$, must be tangent to the price curve and consequently to each other. Now define buyer and seller types $x^{\prime \prime}$ and $y^{\prime \prime}$ as those that prefer the point $\left(p^{\prime \prime}, \theta^{\prime \prime}\right)$ defined by $p^{\prime \prime}=p\left(\theta^{\prime \prime}\right)$, where $p^{\prime \prime}>p^{\prime}$ and $\theta^{\prime \prime}<\theta^{\prime}$. It is not hard to show that $\left(x^{\prime \prime}, y^{\prime \prime}\right)>\left(x^{\prime}, y^{\prime}\right)$-i.e., agents with higher demand and supply prices choose the market with the higher $p$ and lower $\theta .^{12}$

To say more about the equilibrium, first note that the equality of the flow of buyers and sellers into every submarket requires

$$
b\left[F(x)-F\left(R_{b}\right)\right]=s G(y) \quad \forall y \in\left[\underline{y}, R_{s}\right] \quad \text { with } b\left[1-F\left(R_{b}\right)\right]=s G\left(R_{s}\right)
$$

which implies an increasing relationship between $x$ and $y$. At the same time, the tangency condition

$$
\frac{m^{\prime}(\theta)\left[r(p-y)+c_{s}\right]}{r+m(\theta)}=\frac{\left[m(\theta)-\theta m^{\prime}(\theta)\right]\left[r(x-p)+c_{b}\right]}{r \theta+m(\theta)}
$$

implies a decreasing relationship between $x$ and $y$ for every pair $(p, \theta)$. These two equations uniquely identify the assignment of buyer and seller types in the $(p, \theta)$ submarket. We write

${ }^{12}$ Suppose otherwise. Specifically, if there were a buyer of type $x^{\prime \prime}<x^{\prime}$ that prefers $\left(p^{\prime \prime}, \theta^{\prime \prime}\right)$ over $\left(p^{\prime}, \theta^{\prime}\right)$, then from $(29)$

$$
U_{b}\left(x^{\prime}, p^{\prime}, \theta^{\prime}\right)=\frac{m\left(\theta^{\prime}\right)\left(x^{\prime}-p^{\prime}\right)-\theta^{\prime} c_{b}}{r \theta^{\prime}+m\left(\theta^{\prime}\right)}>U_{b}\left(x^{\prime}, p^{\prime \prime}, \theta^{\prime \prime}\right)=\frac{m\left(\theta^{\prime \prime}\right)\left(x^{\prime}-p^{\prime \prime}\right)-\theta^{\prime \prime} c_{b}}{r \theta^{\prime \prime}+m\left(\theta^{\prime \prime}\right)}
$$

and

$$
U_{b}\left(x^{\prime \prime}, p^{\prime}, \theta^{\prime}\right)=\frac{m\left(\theta^{\prime}\right)\left(x^{\prime \prime}-p^{\prime}\right)-\theta^{\prime} c_{b}}{r \theta^{\prime}+m\left(\theta^{\prime}\right)}>U_{b}\left(x^{\prime \prime}, p^{\prime \prime}, \theta^{\prime \prime}\right)=\frac{m\left(\theta^{\prime \prime}\right)\left(x^{\prime \prime}-p^{\prime \prime}\right)-\theta^{\prime \prime} c_{b}}{r \theta^{\prime \prime}+m\left(\theta^{\prime \prime}\right)}
$$

Subtracting the first from the second inequality and collecting terms, we have

$$
\left[\frac{m\left(\theta^{\prime}\right)}{r \theta^{\prime}+m\left(\theta^{\prime}\right)}-\frac{m\left(\theta^{\prime \prime}\right)}{r \theta^{\prime \prime}+m\left(\theta^{\prime \prime}\right)}\right]\left(x^{\prime}-x^{\prime \prime}\right)>0
$$

Because $m(\theta) / \theta$ is decreasing in $\theta$ and $\theta^{\prime \prime}<\theta^{\prime}$, the first term is negative, so the inequality contradicts the supposition $x^{\prime \prime}<x^{\prime}$. An analogous argument implies that $y^{\prime \prime}>y^{\prime}$, and this establishes the claim. 




FiguRE 3

EQUILIBRIUM MARKET SEGMENTATION

$$
x=f\left[p(\theta), \theta, R_{b}\right] \quad \text { and } \quad y=g\left[p(\theta), \theta, R_{b}\right]
$$

where $f(\cdot)$ and $g(\cdot)$ are implicitly defined by (41) and (42). Note that $f(\cdot)$ is increasing and $g(\cdot)$ is decreasing in $R_{b}$.

Summarizing what has been established so far, $p(\theta)$ must be downward sloping, and there is a unique $(x, y)$ associated with each submarket $\theta$, given by (43). To characterize $p(\theta)$ further, substitute $y$ from (43) into the tangency condition $\left.\frac{d p}{d \theta}\right|_{U_{s}}=p^{\prime}(\theta)$ to yield

$$
p^{\prime}(\theta)=-\frac{m^{\prime}(\theta)\left\{r\left[p(\theta)-g\left(p(\theta), \theta, R_{b}\right)\right]+c_{s}\right\}}{m(\theta)[r+m(\theta)]}
$$

This is an ordinary first-order differential equation. We represent its general solution by

$$
p=p(\theta)=p_{0}+h\left(\theta-\theta_{0}, R_{b}\right)
$$

where $h\left(0, R_{b}\right)=0, \partial h / \partial \theta<0$, and $\partial h / \partial R_{b}>0$ by (44), the fact that $g(\cdot)$ is decreasing in $R_{b}$, and the boundary conditions that follow.

The boundary conditions will determine the lowest and highest prices, $p_{0}$ and $p_{1}$, together with the associated $\theta_{0}$ and $\theta_{1}$. The first thing to note is that since buyers with higher $x$ and sellers with higher $y$ go to submarkets with higher prices, in particular the marginal buyer $x=R_{b}$ goes to the submarket with price $p_{0}$ and the marginal seller $y=R_{s}$ goes to the submarket with price $p_{1}$. By (29) and (30), this implies that $R_{b}=p_{0}+\theta_{0} c_{b} / m\left(\theta_{0}\right)$ and $R_{s}=p_{1}-c_{s} / m\left(\theta_{1}\right)$. Inserting these conditions into (41) yields 


$$
b\left[1-F\left(p_{0}+\frac{\theta_{0} c_{b}}{m\left(\theta_{0}\right)}\right)\right]=s G\left(p_{1}-\frac{c_{s}}{m\left(\theta_{1}\right)}\right)
$$

Next, note that (45) implies

$$
p_{1}=p_{0}+h\left(\theta_{1}-\theta_{0}, p_{0}+\frac{\theta_{0} c_{b}}{m\left(\theta_{0}\right)}\right)
$$

Finally, (42) evaluated at $p_{0}$ implies

$$
\begin{aligned}
r\left(p_{0}-\underline{y}\right)+c_{s} & =\frac{\left[r+m\left(\theta_{0}\right)\right]\left[m\left(\theta_{0}\right)-\theta_{0} m^{\prime}\left(\theta_{0}\right)\right]\left[r\left(R_{b}-p_{0}\right)+c_{b}\right]}{m^{\prime}\left(\theta_{0}\right)\left[r \theta_{0}+m\left(\theta_{0}\right)\right]} \\
& =\frac{\left[r+m\left(\theta_{0}\right)\right]\left[m\left(\theta_{0}\right)-\theta_{0} m^{\prime}\left(\theta_{0}\right)\right] c_{b}}{m^{\prime}\left(\theta_{0}\right) m\left(\theta_{0}\right)}
\end{aligned}
$$

and evaluated at $p_{1}$ implies

$$
\begin{aligned}
r\left(\bar{x}-p_{1}\right)+c_{b} & =\frac{m^{\prime}\left(\theta_{1}\right)\left[r \theta_{1}+m\left(\theta_{1}\right)\right]\left[r\left(p_{1}-R_{s}\right)+c_{s}\right]}{\left[r+m\left(\theta_{1}\right)\right]\left[m(\theta)-\theta m^{\prime}(\theta)\right]} \\
& =\frac{m^{\prime}\left(\theta_{1}\right)\left[r \theta_{1}+m\left(\theta_{1}\right)\right] c_{s}}{\left[m\left(\theta_{1}\right)-\theta m^{\prime}\left(\theta_{1}\right)\right] m\left(\theta_{1}\right)}
\end{aligned}
$$

These four equations determine $\left(p_{0}, \theta_{0}\right)$ and $\left(p_{1}, \theta_{1}\right)$.

3.3. Three More Results. Summarizing, we have the following results:

Proposition 4. The sets of steady-state competitive search equilibrium and steady-state Pareto efficient assignments of buyer and seller types to submarkets are equivalent.

Proof. Obvious from the above discussion.

Proposition 5. In every submarket, the seller's and buyer's shares of realized match surplus, respectively, equal the elasticities of the matching function with respect to the stocks of participating buyers and sellers; i.e., the Hosios condition holds.

Proof. Obvious from (40).

Proposition 6. A unique competitive search equilibrium exists for all $r$ sufficiently small.

PROOF. In the case of $r=0$, the price function degenerates to the point

$$
p_{1}=p_{0}=p=\left(1-\frac{\theta m^{\prime}(\theta)}{m(\theta)}\right) R_{b}+\left(\frac{\theta m^{\prime}(\theta)}{m(\theta)}\right) R_{s} \quad \text { and } \quad \theta_{1}=\theta_{0}=\theta
$$

where $\left(R_{b}, R_{s}, \theta\right)$ is the unique solution to 


$$
\begin{aligned}
& \frac{m^{\prime}(\theta)}{m(\theta)-\theta m^{\prime}(\theta)}=\frac{c_{b}}{c_{s}} \\
& R_{b}-R_{s}=\frac{c_{b} \theta+c_{s}}{m(\theta)} \\
& b\left[1-F\left(R_{b}\right)\right]=s G\left(R_{s}\right)
\end{aligned}
$$

Given the continuity of the system (46)-(49) with respect to $r$, we need only demonstrate that these equations have at most one solution when $r$ is sufficiently small. The strict concavity of $m(\theta)$ implies that the solution to (48), call it $p_{0}\left(\theta_{0}\right)$, and the solution to (49), $p_{1}\left(\theta_{1}\right)$, are both strictly increasing for all $r>0$ sufficiently small. Hence,

$$
b\left[1-F\left(p_{0}\left(\theta_{0}\right)+\frac{\theta_{0} c_{b}}{m\left(\theta_{0}\right)}\right)\right]=s G\left(p_{1}\left(\theta_{1}\right)-\frac{c_{s}}{m\left(\theta_{1}\right)}\right)
$$

implicitly defines a decreasing functional relation between $\theta_{0}$ and $\theta_{1}$, while the relation defined by

$$
p_{1}\left(\theta_{1}\right)-p_{0}\left(\theta_{0}\right)=h\left(\theta_{1}-\theta_{0}, p_{0}+\frac{\theta_{0} c_{b}}{m\left(\theta_{0}\right)}\right)
$$

is strictly decreasing given the properties of $h(\cdot)$. The unique solution defines the two endpoints $\left[p_{0}\left(\theta_{0}\right), \theta_{0}\right]$ and $\left[p_{1}\left(\theta_{1}\right), \theta_{1}\right]$ that solve the larger system.

\section{CONCLUSION}

It is our contention that we have learned quite a lot about price determination and efficiency through this exercise. First, the framework provides a simple but compelling story about how prices and quantities are determined in decentralized markets, and why this is approximated by the standard market clearing solution when transaction costs are small. Second, when the conditions for a good approximation do not hold, the search equilibrium price and quantity are not those associated with the intersection of the usual supply and demand curves. Moreover, there are in steady-state stocks of buyers and sellers waiting to exchange-one could say that these markets do not clear either in appearance or in fact. Now, this does not provide a prima facie case for market failure, given transaction costs exist. However, search equilibria do not generally minimize transaction costs when prices are determined ex post according to some arbitrary bargaining solution. To obtain efficiency, a complete set of markets is needed that offers traders the opportunity to shorten the time required to find a trading partner at cost in terms of the price. A question that remains is, who provides these markets? We offered a story about market makers, but certainly more work should be done on this dimension. Sattinger (2000) provides one such attempt.

\section{REFERENCES}

Acemoglu, D., and R. Shimer, "Holdups and Efficiency with Search Frictions," International Economic Review 40 (1999), 827-50. 
Burdett, K., R. Wright, AND S. Shi, "Pricing and Matching with Frictions," Journal of Political Economy, 109 (2001), 1060-85.

Diamond, P. A., "Mobility Costs, Frictional Unemployment, and Efficiency," Journal of Political Economy 89 (1981), 798-812.

GALE, D., "Limit Theorems for Markets with Sequential Bargaining," Journal of Economic Theory 43 (1987), 20-54.

Greenwald, B., AND J. E. Stiglitz, "Pareto Inefficiency of Market Economies: Search and Efficiency Wage Models," American Economic Review Papers and Proceedings 78 (1988), 351-56.

Hosios, A. J., "On the Efficiency of Matching and Related Models of Search and Unemployment," Review of Economic Studies 57 (1990), 279-98.

Julien, B., J. Kennes, AND I. KInG, "Bidding for Labor," Review of Economic Dynamics 3 (2000), 619-49.

Moen, E. R., “Competitive Search Equilibrium,”'Journal of Political Economy 105 (1997), 385-411.

Mortensen, D. T., "The Matching Process as a Noncooperative Bargaining Game,'” in J. J. McCall, ed., The Economics of Information and Uncertainty (Chicago: University of Chicago Press, 1982), 233-54.

Osborne, M. J., And A. Rubinstein, Bargaining and Markets (New York: Academic Press, 1990).

PIssARIDES, C. A., Equilibrium Unemployment Theory (Oxford: Basil Blackwell, 1990).

Rubinstein, A., AND A. Wolinsky, "Equilibrium in a Market with Sequential Bargaining," Econometrica 53 (1985), 1133-50.

SAtTinger, M., "Brokers and the Equilibrium Price Function," working paper, 2000.

SHIMER, R., "Contracts in a Frictional Labor Market," working paper, 1995. 